\title{
LA CANCIÓN INFANTIL COMO CANCIÓN ESCOLAR. PROPUESTA PARA UNA FICHA DE ANÁLISIS
}

\section{Nursery songs in the classroom. Proposal for an analysis sheet}

María Consuelo de la Vega Sestelo Universidad de Salamanca. Dpto. de Didáctica de la Expresión Física, Plástica y Musical

Correo-e: vegasestelo@usal.es

Recepción: ro de julio de 2014

Envío a informantes: 20 de septiembre de 2014

Aceptación definitiva: 2I de enero de 2015

Biblid. [0214-3402 (2015) (II época) n. ${ }^{\circ}$ 21; 193-206]

Resumen: La canción infantil es uno de los elementos básicos sobre los que se fundamenta la educación musical en la enseñanza primaria en España. Necesitamos de un recurso de análisis, desde el punto de vista del docente, que sirva de herramienta de elección de las distintas canciones que van a ser utilizadas en el aula dirigidas a distintos niveles, cursos, edades de alumnos, etc. Se realiza una propuesta de organización de este análisis estableciendo un esquema de actuación y ofreciendo las distintas categorías que para cada apartado se proponen. Tendremos en cuenta aspectos que intervienen en la construcción de una canción infantil como una canción escolar: musicales, lingüísticos, didácticos, metodológicos, culturales, etc. Por otro lado, el estudio de las canciones en las distintas épocas históricas ofrecerá una posibilidad de concatenación histórica sobre la evolución que en su construcción y en su utilización educativa ofrecen estas pequeñas obras musicales. En su faceta artística estas canciones podrán ser interpretadas y reinterpretadas con nuevas y actuales concepciones didácticas.

Palabras clave: canción infantil; canción escolar; educación musical; enseñanza primaria; análisis educativo-musical; categoría de análisis.

ABSTRACT: Nursery songs are one of the basic pillars of musical education in primary schools in Spain. Teachers need analytical resources providing them with tools to select the choice of songs they will use in the classroom for each different level, school year, age group, etc. This paper offers a proposal for the organisation of such an analysis, establishing an action plan and suggesting the categories to be included in each section. The following aspects relevant to the composition of nursery songs as classroom songs are taken into consideration: musical, linguistic, didactic, methodological, cultural, etc. Moreover, the study of such songs over a range of historical periods provides an 
opportunity for establishing a historical sequence for the evolution of these minor musical works from the point of view of their composition and their educational use. With regard to their artistic content, these songs can be interpreted and reinterpreted under new and current educational ideologies.

KEY WORDS: nursery song; classroom song; musical education; primary education; musical education training; analytical category.

\section{I. ¿Por qué una canción infantil se convierte en una canción escolar? ¿Qué se puede enseñar?}

$\mathrm{L}$

A EDUCACIÓN ARTÍSTICA Y EN CONCRETO LA EDUCACIÓN MUSICAL SOn aspectos de la educación integral de la persona que han contemplado los distintos planes de estudio de la enseñanza primaria ya desde finales del siglo xIx. Este tipo de educación no es algo nuevo en la estructuración de lo que la sociedad considera necesario enseñar a las generaciones que van naciendo, ya los griegos, con Platón (ed. I998), como exponente fundamental, otorgaban a la música un poder pedagógico y de influencia para desarrollar en las personas valores sociales, políticos, estéticos y personales necesarios para afrontar la vida y la realidad que les tocaría vivir.

Nos centraremos en una época más reciente, después del nacimiento de los sistemas nacionales de educación, organigramas que se fueron construyendo a lo largo del siglo xIX y que fueron introduciendo contenidos educativos, objetivos y valores acordes con las demandas sociales de cada época, atendiendo a la evolución que cada sociedad iba experimentando. En I90I, se estructura un plan de estudios de la primera enseñanza pública para todas las escuelas de la nación. En este plan se incluye, por primera vez, el Canto como materia (puesta en marcha de forma experimental en las escuelas graduadas anejas a las Escuelas Normales en I899), especificando, al igual que en las demás, que estará presente en los tres grados en que se divide la enseñanza diferenciándose en cada curso únicamente en la amplitud del programa y en el carácter pedagógico y duración de los ejercicios (Real Decreto de 26 de octubre de I9or, art. $3 .^{\circ} \mathrm{y}^{\circ} \cdot^{\circ}$.

Con el Plan de estudios de I90 llegamos a 1945, con todos los avatares que en tantos años sufrió la sociedad española, política y culturalmente. En ese año, en pleno periodo franquista, se promulga la Ley sobre Enseñanza Primaria donde se sigue incluyendo en el conjunto de las asignaturas a la Música y Canto dentro de las materias complementarias (Ley de i7 julio de 1945, art. 37).

En los sucesivos planes de estudios que fueron desarrollo de las Leyes de Educación en España, como la Ley General de Educación de 1970, la Ley Orgánica de Ordenación General del Sistema Educativo (LOGSE) de 1990 y la Ley Orgánica de Educación (LOE) de 2006 se ha incluido a la enseñanza de la música dentro de la Educación artística junto a la plástica y en algunas ocasiones a la dramatización como lenguajes artísticos. En la última ley aprobada en nuestro país, la Ley Orgánica para la Mejora de la Calidad Educativa (LOMCE, 2OI3), se sigue considerando necesaria la adquisición del sentido artístico en la formación inicial de las personas (punto siete). El Real Decreto (20I4, art. 8) que desarrolla el currículo básico de Educación Primaria deja a la regulación y programación de la oferta educativa de las Comunidades Autónomas la estructuración, entre otras, de la Educación artística, en los centros docentes. La Comunidad 
de Castilla y León ha establecido que el alumnado de este nivel educativo curse como asignatura específica la Educación Artística, en cada uno de los cursos (Orden EDU/519, 20I4, art. 9).

Una vez que hemos dejado constancia de incluir a la Música y el Canto en la educación primaria a lo largo de todo el siglo xx y lo que nos ocupa del xxI en nuestras escuelas, podemos referirnos al material que se encuentra al alcance de maestros para poder conseguir los objetivos establecidos en cada momento para esta área de conocimiento, podemos referirnos ya a la canción infantil como una herramienta metodológica que incluye dentro de sí misma rasgos de sencillez y a la vez de complejidad de los contenidos necesarios en la enseñanza, rasgos estéticos y culturales, aspectos lúdicos y académicos que facilitan su aprendizaje y su utilización didáctica. Podemos citar aquí a importantes pedagogos musicales como Orff, Dalcroze, Kodaly, Willems o Ward que desarrollaron su actividad pedagógica y musical utilizando la canción infantil como eje conductor de las actividades musicales con los niños. Ellos son el germen que sirve de base para que otros pedagogos de la educación musical como Martínez Torner, Benedito, Borguñó, Rogelio Villar, Llongueras o Salvador Martí considerasen a la canción como elemento fundamental en la educación musical de los niños. En palabras de Willems (1976, $4{ }^{\text {a }}$ ed.):

Nunca se insistirá bastante sobre la importancia de los cantos en la educación musical de los niños. Los cantos constituyen una actividad sintética; engloban la sensibilidad y el ritmo, y además sugieren el acorde y hacen presentir las funciones sintéticas (p. 53).

Es de justicia histórica dejar constancia aquí de los planteamientos educativos de la Institución Libre de Enseñanza y lo que para ellos suponía el empleo del canto coral; en palabras de Leticia Sánchez (2009) podemos resumirlos como: educación del sentimiento estético a través de la práctica activa de la música; herramienta útil para mediatizar otros aprendizajes; estimula la sociabilidad y la capacidad creativa del individuo así como el trabajo en comunidad; y forma al cuerpo, a la razón y al sentimiento.

En la canción se unen letra y música. Esta combinación ofrece múltiples posibilidades de utilización pedagógica, de recursos didácticos, de fórmulas de aprendizaje muy importantes no solo en el estudio de la música, sino como herramienta metodológica en el aprendizaje de otras materias del currículum. Murray Schafer (1970) lo refleja en la siguiente deducción lógica:

Lenguaje es comunicación a través de combinaciones simbólicas de fonemas llamados palabras.

Música es comunicación a través de combinaciones de sonidos y de objetos sonoros. Luego lenguaje es sonido como significado. Música es sonido como sonido.

La canción escolar ofrece la posibilidad de actuaciones pedagógicas que se enmarcan en conceptos de libertad, creatividad, improvisación, descubrimiento, aprendizaje, experimentación y participación activa que hace que este material pueda ser utilizado en múltiples aspectos de la educación, musical y no musical, con objetivos englobados en la educación integral de los individuos que les ayudará a enfrentarse a la vida y a la resolución, de forma creativa, de los problemas que esta plantea. Hemsy de Gainza (2002) hace referencia a ello cuando nos comenta que la práctica musical creativa, la experimentación, el descubrimiento personal y grupal constituyen las 
condiciones naturales de la libertad para crecer y desarrollarse. Continúa diciendo que la creatividad es, a la vez, punto de partida y meta, para las relaciones educando/ maestro, relaciones con el proceso y las formas de acción pedagógica.

\section{Propuesta de ficha de análisis de una canción escolar}

Un canción infantil analizada como una canción escolar tiene varias vertientes para su estudio: organización y estructura del documento, análisis musical, instrumental, sobre el texto, educativo, social, cultural e histórico. En esta parte estableceremos una estructura por cada apartado para posteriormente organizar las categorías necesarias para un estudio comparativo, histórico, analítico, didáctico, etc. Es una propuesta que deberá ser adaptada a la época de estudio, autores, situaciones y circunstancias didácticas y educativas que cada investigador, estudioso o docente, que debe elegir la canción como material de trabajo, estime oportuno.

\section{Ficha de análisis}

I. Organización y estructura del documento donde se encuentra la canción.

I.I. Autor: referido a la música.

I.2. Año de publicación: centra la época histórica en que se encuentra la canción.

I.3. Referencias: nos informa sobre la constatación o no de la utilización práctica de este material.

I.4. Localización: ubicación física del material para su uso.

I.5. Estructura: división y formato utilizado.

2. Análisis musical.

2.I. Melódico de las canciones.

2.I.I. Tonalidades: conocimiento del uso de las más frecuentes por los compositores.

2.I.2. Alteraciones accidentales: utilización o no y forma de aparición.

2.I.3. Intervalos melódicos: analizando horizontalmente la melodía de la canción.

2.I.4. Intervalos armónicos: en canciones a dos o más voces con un análisis vertical entre ellas.

2.I.5. Extensión: número de compases totales de la canción.

2.I.6. Ámbito: nota más grave y más aguda en la melodía.

2.2. Rítmico.

2.2.I. Compases: más frecuentes.

2.2.2. Figuras y silencios: grupos rítmicos en subdivisión binaria utilizados.

2.2.3. Figuras y silencios: grupos rítmicos en subdivisión ternaria utilizados.

2.2.4. Notas de adorno: solo se reseñarán las empleadas en la melodía de la canción.

2.3. Armónico.

2.3.I. Cambios de tonalidad y modulaciones: cambio de tonalidad como cambio de armadura y modulación como variación tonal o flexiones modulantes en el transcurso del texto musical.

2.3.2. Cadencias: solo se analizan las finales al ser obras de pequeño formato. 
2.4. Formal.

2.4.I. Formas músico-elementales: clasificación de las más habituales.

2.4.2. Comienzo de la melodía.

2.4.3. Final de la melodía.

2.4.4. Extensión de las frases musicales: número de compases por frase musical.

2.4.5. Estructura de las frases musicales: forma de construcción de cada frase.

2.4.6. Regularidad de las frases musicales: estructura regular o irregular en la composición de cada frase musical.

2.5. Agógico y dinámico, articulación y acentuación.

2.5.I. Tempo: términos utilizados en función de intervalos de velocidades.

2.5.2. Carácter: términos empleados según la expresividad.

2.5.3. Términos agógicos.

2.5.4. Términos dinámicos.

2.5.5. Articulación y acentuación: signos más frecuentes.

2.5.6. Otros términos.

3. Análisis del acompañamiento.

3.I. Acompañamiento instrumental: solo se registra inclusión o no.

3.2. Acompañamiento corporal: solo se registra inclusión o no.

4. Análisis del texto.

4.I. Idioma del texto.

4.2. Autores del texto: pueden coincidir o no con el autor de la música, o ser anónimos.

4.3. Estructura del texto: obtención de datos que afectan a la estructura musical.

4.4. Adecuación texto y música: análisis de las acentuaciones musicales y prosódicas.

4.5. Onomatopeyas: análisis en texto y música.

4.6. Temas de los textos: nos ofrecen información sobre contenidos y objetivos educativos en la utilización didáctica de la canción.

5. Análisis educativo y didáctico.

5.I. Contenido didáctico y metodológico: aspectos que incluye el autor sobre la forma de enseñarla.

5.2. Tipología de los alumnos: características de los destinatarios del aprendizaje de la canción.

6. Análisis histórico, social y cultural.

6.I. Histórico: información sobre la época de creación de la canción y sus objetivos educativos.

6.2. Social: utilización social de la canción infantil.

6.3. Cultural: intervención de la canción infantil en la evolución cultural de una sociedad.

\section{Organización de categorías de análisis}

Para cada apartado expuesto establecemos categorías de análisis que exponemos de forma resumida en las siguientes tablas. Estas categorías nos ayudan a la agrupación de las canciones, a su estudio comparativo, a su estudio musical, histórico y a una catalogación necesaria para la investigación educativa y musical, para ofrecer al docente colecciones de canciones con determinadas características musicales, educativas y culturales para poder ser utilizadas en la escuela. 
Estas categorías se han elaborado después de analizar 782 canciones infantiles y escolares publicadas o utilizadas en España entre 1900 y 1936 (Vega Sestelo, 2013). Obedecen a los elementos musicales y no musicales encontrados en estos textos que pueden ser extrapolados al análisis de una canción en cualquier momento desde el siglo xix a la actualidad, donde la Teoría de la Música contiene aspectos básicos constantes en gran parte de sus contenidos.

Tanto la ficha de análisis como las categorías establecidas son susceptibles de mejora o adaptación dependiendo de la época de estudio, de los autores tratados, de los enfoques concretos de una investigación o de los enfoques concretos de las necesidades docentes para el empleo de canciones infantiles en el aula.

Utilizamos la misma numeración y apartados que en la ficha propuesta para facilitar la lectura de este trabajo:

\section{Organización y estructura del documento}

- Objetivos del apartado:

Se pretende conocer todos aquellos aspectos referidos al contexto externo de construcción de la obra, de la delimitación histórica y geográfica, de la localización para su estudio, de las referencias de utilización y de los autores que nos acercarán a un conocimiento del momento en el que fueron creadas, de su presentación a la sociedad y profesionales que podrían utilizarlas y de los aspectos educativos de una época determinada y su influencia o valoración por los distintos sectores implicados en ellos.

Tabla i. Categorías de análisis para el análisis de la organización y estructura del documento

\begin{tabular}{|c|c|}
\hline \multicolumn{2}{|r|}{ I. Organización y estructura del documento } \\
\hline Apartados de análisis & Categorías de análisis \\
\hline I.I. Autor & $\begin{array}{l}\text { a) Procedencia de los autores } \\
\text { b) Formación musical de los autores } \\
\text { c) Relación con la docencia } \\
\text { d) Número de canciones compuestas } \\
\text { e) Canciones sin autor conocido }\end{array}$ \\
\hline I.2. Año de publicación & $\begin{array}{l}\text { a) Siglo XIx hasta I878 } \\
\text { b) De } 1878 \text { a I898 } \\
\text { c) De } 1898 \text { a } 1936 \\
\text { d) De } 1936 \text { a I939 } \\
\text { e) Periodo franquista hasta } 1978 \\
\text { f) De I978 hasta año } 2000 \\
\text { g) Siglo XXI }\end{array}$ \\
\hline I.3. Referencias & $\begin{array}{l}\text { a) Canciones con constancia de haber sido utilizadas o propuestas } \\
\text { didácticamente } \\
\text { b) Canciones sin referencias }\end{array}$ \\
\hline ı.4. Localización & $\begin{array}{l}\text { a) Noreste de España } \\
\text { b) Noroeste de España } \\
\text { c) Madrid } \\
\text { d) Zona centro } \\
\text { e) Sur de España }\end{array}$ \\
\hline I.5. Estructura & $\begin{array}{l}\text { a) Número de canciones en la publicación } \\
\text { b) Inclusión de parte teórica o metodológica } \\
\text { c) Inclusión de aspectos de movimiento y danza } \\
\text { d) Inclusión de ilustraciones }\end{array}$ \\
\hline
\end{tabular}

Fuente: Elaboración propia. 


\section{Análisis musical}

- Objetivos del apartado:

Se pretende analizar los siguientes aspectos musicales que intervienen en la composición de estas obras. De esta forma podremos utilizarlas, en primer lugar, en relación a los contenidos de aprendizaje de un determinado curso o grupos de alumnos; y, en segundo lugar, como herramientas metodológicas en materias o asignaturas, sabiendo que el aprendizaje de la canción y su asimilación está al alcance educativo de los alumnos a los que va destinada la elección.

TABLA II. Categorías de análisis para el análisis melódico

\begin{tabular}{|c|c|}
\hline \multicolumn{2}{|r|}{ 2.1. Análisis melódico } \\
\hline Apartados de análisis & Categorías de análisis \\
\hline 2.I.I. Tonalidades & $\begin{array}{l}\text { a) Ninguna alteración en la armadura } \\
\text { b) Una y dos alteraciones en la armadura } \\
\text { c) Tres y cuatro alteraciones en la armadura } \\
\text { d) Cinco o más alteraciones en la armadura } \\
\text { e) Canciones modales }\end{array}$ \\
\hline 2.I.2. Alteraciones accidentales & $\begin{array}{l}\text { a) Con alteraciones accidentales } \\
\text { b) Sin alteraciones accidentales }\end{array}$ \\
\hline 2.I.3. Intervalos melódicos & $\begin{array}{l}\text { a) Intervalos: } 4 \cdot \cdot^{\mathrm{a}} \text { y } 5 \cdot{ }^{\mathrm{a}} \text { Justas; } 2 .^{\mathrm{a}}, 3 \cdot{ }^{\mathrm{a}} \text { y } 6 \cdot^{\mathrm{a}} \text { Mayores y menores } \\
\text { b) Intervalos aumentados y disminuidos } \\
\text { c) Intervalos de } 7 \cdot{ }^{\mathrm{a}} \text { Mayores y menores } \\
\text { d) Intervalo de } 8 \cdot{ }^{\mathrm{a}} \text { Justa } \\
\text { e) Intervalos compuestos }\end{array}$ \\
\hline 2.I.4. Intervalos armónicos & $\begin{array}{l}\text { a) Categoría común a todas: } 3 \cdot{ }^{\mathrm{a}} \text { y } 6 \cdot{ }^{\mathrm{a}} \mathrm{M} \text { y m } \\
\text { b) Consonancias perfectas (Justos) } \\
\text { c) Disonancias absolutas }\left(2 \cdot{ }^{\mathrm{a}} \text { y } 7 \cdot \cdot^{\mathrm{a}} \mathrm{M} \text { y m) }\right. \\
\text { d) Semiconsonancias }\left(4 \cdot{ }^{\mathrm{a}} \mathrm{A} \text { y } 5 \cdot{ }^{\mathrm{a}} \mathrm{d}\right)\end{array}$ \\
\hline 2.I.5. Extensión & $\begin{array}{l}\text { a) Canciones de hasta I2 compases } \\
\text { b) Canciones entre } 13 \text { y } 24 \text { compases } \\
\text { c) Canciones entre } 25 \text { y } 48 \text { compases } \\
\text { d) Canciones de } 49 \text { o más compases }\end{array}$ \\
\hline 2.I.6. Ámbito & $\begin{array}{l}\text { a) } \mathrm{De}_{\mathrm{Do}_{4}} \mathrm{a} \mathrm{La}_{4} \\
\text { b) } \mathrm{DeSi}_{3} \mathrm{a} \mathrm{Do}^{5} \\
\text { c) De } \mathrm{La}_{3} \mathrm{a} \mathrm{Mi}_{5} \\
\text { d) Superan los límites anteriores }\end{array}$ \\
\hline
\end{tabular}

Fuente: Elaboración propia.

TABla iII. Categorías de análisis para el análisis rítmico

\begin{tabular}{|l|l|}
\hline \multicolumn{2}{|c|}{ 2.2. Análisis rítmico } \\
\hline \multicolumn{1}{|c|}{ Apartados de análisis } & \multicolumn{1}{c|}{ Categorías de análisis } \\
\hline 2.2.I. Compases & \begin{tabular}{l}
\multicolumn{1}{c|}{ a) Subdivisión binaria: $4 / 4,3 / 4,2 / 4,2 / 2,2 / 8$ y $3 / 8$} \\
b) Subdivisión ternaria: $6 / 8,9 / 8 \mathrm{y} \mathrm{I} 2 / 8$ \\
\\
\end{tabular} \\
& $\begin{array}{l}\text { c) Otros compases: } 5 / 8 \\
\text { d) Canciones a modo de recitativo } \\
\text { e) Canciones con cambio de compás }\end{array}$ \\
\hline
\end{tabular}




\begin{tabular}{|c|c|}
\hline $\begin{array}{l}\text { 2.2.2. Figuras y silencios: grupos } \\
\text { rítmicos en subdivisión binaria }\end{array}$ & $\begin{array}{l}\text { a) Formulaciones rítmicas básicas } \\
\text { b) Grupos rítmicos sincopados } \\
\text { c) Grupos con figuras a contratiempo } \\
\text { d) Grupos con puntillos } \\
\text { e) Grupos con trabajo inicial de semicorcheas } \\
\text { f) Grupos con silencios de semicorchea } \\
\text { g) Grupos que utilizan la ligadura de prolongación } \\
\text { h) Grupos rítmicos de valoración especial } \\
\text { i) Grupos con fusas }\end{array}$ \\
\hline $\begin{array}{l}\text { 2.2.3. Figuras y silencios: grupos } \\
\text { rítmicos en subdivisión ternaria }\end{array}$ & $\begin{array}{l}\text { a) Formulaciones rítmicas básicas } \\
\text { b) Grupos rítmicos que incluyen semicorcheas } \\
\text { c) Grupos que incluyen silencio de corchea } \\
\text { d) Grupos con puntillos de prolongación } \\
\text { e) Grupos con ligaduras de prolongación } \\
\text { f) Grupos con silencios de semicorchea } \\
\text { g) Grupos que producen contratiempos } \\
\text { h) Grupos rítmicos que incluyen síncopas } \\
\text { i) Grupos con fusas }\end{array}$ \\
\hline 2.2.4. Notas de adorno & $\begin{array}{l}\text { a) Apoyatura } \\
\text { b) Mordente de I nota } \\
\text { c) Mordente de } 2 \text { notas } \\
\text { d) Mordente de } 3 \text { notas }\end{array}$ \\
\hline
\end{tabular}

Fuente: Elaboración propia.

TABLA Iv. Categorías de análisis para el análisis armónico

\begin{tabular}{|c|c|}
\hline \multicolumn{2}{|r|}{ 2.3. Análisis armónico } \\
\hline Apartados de análisis & Categorías de análisis \\
\hline $\begin{array}{l}\text { 2.3.I. Cambios de tonalidad y } \\
\text { modulaciones }\end{array}$ & $\begin{array}{l}\text { a) Al tono homónimo } \\
\text { b) Al tono relativo } \\
\text { c) Al tono de la dominante } \\
\text { d) A tonos vecinos } \\
\text { e) A tonos lejanos }\end{array}$ \\
\hline 2.3.2. Cadencias finales & $\begin{array}{l}\text { a) Cadencia perfecta } \\
\text { b) Cadencia imperfecta } \\
\text { c) Cadencia plagal } \\
\text { d) Semicadencia a la dominante }\end{array}$ \\
\hline
\end{tabular}

Fuente: Elaboración propia.

TABLA v. Categorías de análisis para el análisis formal

\subsection{Análisis formal}

Apartados de análisis

\section{Categorías de análisis}

2.4.I. Formas músico-elementales a) Lied a una parte (A)

b) Lied a dos partes (A-B)

c) Lied a tres partes (A-B-A)

d) Lied a tres partes (A-B-C)

e) Canon

f) Rondó

g) Otras: canciones irregulares en estructura 


\begin{tabular}{|l|l|}
\hline 2.4.2. Comienzo de la melodía & a) Tético \\
& b) Anacrúsico \\
& c) Acéfalo \\
\hline 2.4.3. Final de la melodía & a) Masculino \\
& b) Femenino \\
\hline 2.4.4. Extensión de las frases & a) Menores de 8 compases \\
& b) De 8 compases \\
& c) Entre 9 y II compases \\
& d) De I2 compases \\
& e) Mayores de I2 compases \\
\hline 2.4.5. Estructura de las frases & a) Binarias \\
& b) Ternarias \\
& c) Cuaternarias \\
& d) Otras: frases sin división, consideradas en un solo discurso melódico \\
\hline 2.4.6. Regularidad de las frases & a) Regulares \\
musicales & b) Irregulares \\
\hline
\end{tabular}

Fuente: Elaboración propia.

TABla vi. Categorías de análisis para el análisis agógico y dinámico, articulación y acentuación

\begin{tabular}{|c|c|}
\hline \multicolumn{2}{|c|}{ 2.5. Análisis agógico y dinámico, articulación y acentuación } \\
\hline Apartados de análisis & Categorías de análisis \\
\hline 2.5.I. Tempo & $\begin{array}{l}\text { a) Velocidades de hasta } 66 \text { pulsos por minuto } \\
\text { b) Velocidades entre } 66 \text { y } 76 \text { pulsos por minuto } \\
\text { c) Velocidades entre } 76 \text { y I20 pulsos por minuto } \\
\text { d) Velocidades entre I20 y I68 pulsos por minuto } \\
\text { e) Velocidades mayores de I } 68 \text { pulsos por minuto } \\
\text { f) Términos de aires de danza } \\
\text { g) Canciones sin indicación de tempo } \\
\text { h) Canciones con cambios de Aire }\end{array}$ \\
\hline 2.5.2. Carácter & $\begin{array}{l}\text { a) Términos que reflejan expresión de sentimientos } \\
\text { b) Indicaciones que afectan a la velocidad } \\
\text { c) Términos con carácter rítmico } \\
\text { d) Términos que describen una situación } \\
\text { e) Términos que expresan una actitud }\end{array}$ \\
\hline 2.5.3. Términos agógicos & $\begin{array}{l}\text { a) Términos que indican aumento gradual de la velocidad } \\
\text { b) Términos que indican disminución gradual de la velocidad } \\
\text { c) Términos que indican aumento de velocidad (no gradual) } \\
\text { d) Términos que indican disminución de la velocidad (no gradual) } \\
\text { e) Términos que indican restitución del tempo inicial } \\
\text { f) Otros términos }\end{array}$ \\
\hline 2.5.4. Términos dinámicos & $\begin{array}{l}\text { a) } p p p \text { у } p p \\
\text { b) } p \\
\text { c) } m p \\
\text { d) } m f \\
\text { e) } f \\
\text { f) } f f \text { y fff } \\
\text { g) Términos que aumentan gradualmente la intensidad } \\
\text { h) Términos que disminuyen gradualmente la intensidad } \\
\text { i) Reguladores } \\
\text { j) Otros }\end{array}$ \\
\hline
\end{tabular}




\begin{tabular}{|l|l|}
\hline 2.5.5. Articulación y acentuación & a) Ligadura de expresión \\
& b) Coma de respiración \\
& c) Acento \\
& d) Indicaciones de legato \\
& e) Picado \\
& f) Ligado-Picado \\
& g) Subrayado \\
& h) Marcato \\
\hline 2.5.6. Stz y sf \\
\hline
\end{tabular}

Fuente: Elaboración propia.

\section{Análisis del acompañamiento}

- Objetivos del apartado:

Este análisis solo se realiza en función de la inclusión o no de algún tipo de acompañamiento. Su estudio profundo sería objeto de un trabajo específico y concreto para tal fin. Se pretende facilitar la elección de la canción al docente y una primera aproximación a la investigación de los acompañamientos instrumentales y corporales que estas obras pueden tener.

Tabla viı. Categorías de análisis para el análisis del acompañamiento

\begin{tabular}{|l|l|}
\hline \multicolumn{1}{|c|}{ 3. Análisis del acompañamiento } \\
\hline \multicolumn{1}{|c|}{ Apartados de análisis } & \multicolumn{1}{c|}{ Categorías de análisis } \\
\hline $\begin{array}{l}\text { 3.I. Acompañamiento } \\
\text { instrumental }\end{array}$ & $\begin{array}{l}\text { a) Con acompañamiento instrumental } \\
\text { b) Sin acompañamiento instrumental }\end{array}$ \\
\hline 3.2. Acompañamiento corporal & $\begin{array}{l}\text { a) Con acompañamiento corporal } \\
\text { b) Sin acompañamiento corporal }\end{array}$ \\
\hline
\end{tabular}

Fuente: Elaboración propia.

\section{Análisis del texto}

- Objetivos del apartado:

El texto, en una canción escolar, es tan importante como la música. Van unidos en la composición, ninguno destaca o sobresale, siendo ambos de vital importancia. Desde el punto de vista didáctico, el texto aporta datos de intenciones educativas que pueden ser utilizados en el aula. Para una elección apropiada de una canción infantil como escolar, es necesario conocer todo lo relativo al texto. 
TABla viII. Categorías de análisis para el análisis del texto

\begin{tabular}{|l|l|}
\hline \multicolumn{2}{|c|}{ 4. Análisis del texto } \\
\hline \multicolumn{1}{|c|}{ Apartados de análisis } & \multicolumn{1}{c|}{ Categorías de análisis } \\
\hline 4.I. Idioma del texto & $\begin{array}{l}\text { a) Castellano } \\
\text { b) Catalán, Gallego y Euskera } \\
\text { c) Otros }\end{array}$ \\
\hline 4.2. Autores del texto & $\begin{array}{l}\text { a) Canciones donde el autor de la música lo es de la letra } \\
\text { b) Canciones donde el autor de la música lo es de la letra en parte de } \\
\text { ellas } \\
\text { c) Canciones donde el texto corresponde a distinto autor que la música } \\
\text { d) Canciones con textos latinos o de eminentes poetas } \\
\text { e) Canciones con textos populares }\end{array}$ \\
\hline 4.3. Estructura de la composición & $\begin{array}{l}\text { a) Prosa } \\
\text { b) Verso }\end{array}$ \\
\hline 4.4. Adecuación texto y música & $\begin{array}{l}\text { a) Muy buena } \\
\text { b) Buena } \\
\text { c) Regular } \\
\text { d) Mala }\end{array}$ \\
\hline 4.5. Onomatopeyas & $\begin{array}{l}\text { a) Sobre instrumentos musicales } \\
\text { b) Sobre Naturaleza y animales } \\
\text { c) Sobre sonidos de la vida cotidiana } \\
\text { d) Otros }\end{array}$ \\
\hline 4.6. Temas de los textos & $\begin{array}{l}\text { a) La vida en la escuela } \\
\text { b) Enseñanza moral o de preparación para la vida } \\
\text { c) Sobre la Naturaleza } \\
\text { d) Patrióticas } \\
\text { e) Vida cotidiana del niño } \\
\text { f) Religiosas } \\
\text { g) De contenidos de aprendizaje } \\
\text { h) Sobre oficios y profesiones } \\
\text { i) Folklóricas } \\
\text { j) Otras }\end{array}$ \\
\hline
\end{tabular}

Fuente: Elaboración propia.

\section{Análisis educativo y didáctico}

- Objetivos del apartado:

Los objetivos se centran, por un lado, en el conocimiento de las dificultades que la enseñanza y aprendizaje de una determinada canción supone, dependiendo de aspectos metodológicos, tipos de contenidos y dificultades de aprendizaje; por otro lado, nos aporta información sobre sus destinatarios, los alumnos, su formación inicial para poder abordar el aprendizaje de una u otra canción. Desde el punto de vista de la investigación educativa, este análisis nos ayuda a establecer aspectos que relacionen las competencias educativas, los objetivos educativos de un determinado nivel o curso y los contenidos necesarios para llegar a alcanzar estos objetivos y adquirir dichas competencias. 
Tabla ix. Categorías de análisis para el análisis educativo y didáctico

\begin{tabular}{|l|l|}
\hline \multicolumn{1}{|c|}{ 5. Análisis educativo y didáctico } \\
\hline \multicolumn{1}{|c|}{ Apartados de análisis } & \multicolumn{1}{c|}{ Categorías de análisis } \\
\hline $\begin{array}{l}\text { 5.I. Contenido didáctico y } \\
\text { metodológico }\end{array}$ & $\begin{array}{l}\text { a) Principios metodológicos } \\
\text { b) Aspectos didácticos del método } \\
\text { c) Metodología que debe emplear el maestro }\end{array}$ \\
\hline 5.2. Tipología de los alumnos & $\begin{array}{l}\text { a) Canciones para alumnos con preparación musical previa } \\
\text { b) Canciones para alumnos que se inician en el canto }\end{array}$ \\
\hline
\end{tabular}

Fuente: Elaboración propia.

\section{Análisis histórico, social y cultural}

- Objetivos del apartado:

La música es un arte. Como tal está vivo en el tiempo, solo depende de su interpretación y reinterpretación en épocas distintas a su creación. Las canciones escolares, como obras musicales, pueden ser utilizadas en momentos y años distintos para los que fueron creadas. Necesitamos su conocimiento histórico, social y cultural porque nos acerca al conocimiento de los objetivos educativos de dicho periodo de la historia. Su utilización en la época actual dependerá de este conocimiento previo y de su adecuación por parte del docente a los planes de estudio o estructuras educativas que se encuentran en vigor. Desde el punto de vista de la investigación educativa nos ayuda a establecer caminos continuos de conocimiento donde poder estudiar la evolución musical, social y cultural de estas composiciones.

TABLA x. Categorías de análisis para el análisis histórico, social y cultural

\begin{tabular}{|l|l|}
\hline \multicolumn{1}{|c|}{ Apartados de análisis } & \multicolumn{1}{|c|}{ C. Análisis histórico, social y cultural } \\
\hline 6.I. Histórico & $\begin{array}{l}\text { a) Épocáas de análisis } \\
\text { b) Época histórica de su utilización } \\
\text { c) Adecuación a los objetivos educativos de cada etapa histórica }\end{array}$ \\
\hline 6.2. Social & $\begin{array}{l}\text { a) Canciones infantiles destinadas a la escuela } \\
\text { b) Canciones infantiles destinadas al juego } \\
\text { c) Canciones con fines políticos y morales } \\
\text { d) Canciones de transmisión de costumbres } \\
\text { e) Canciones que reflejan características de la época social de su } \\
\text { creación }\end{array}$ \\
\hline 6.3. Cultural & $\begin{array}{l}\text { a) Canciones infantiles de compositores consagrados } \\
\text { b) Canciones que contribuyen al desarrollo cultural de una época } \\
\text { c) Canciones infantiles con fines culturales pero no escolares }\end{array}$ \\
\hline
\end{tabular}

Fuente: Elaboración propia. 


\section{Conclusiones}

I. La canción infantil desde el punto de vista educativo, como canción escolar, es uno de los hilos conductores pedagógico, didáctico y musical de la educación artística en la enseñanza primaria en España.

2. Necesitamos conocer, a través de un análisis riguroso y detallado, las canciones infantiles como fuente de material pedagógico, no solo las canciones que se crean en la actualidad, sino todas aquellas que a lo largo de los años se han ido componiendo y que pueden ser utilizadas como herramientas metodológicas en la enseñanza en la época actual.

3. Consideramos como ayuda inicial ofrecer una estructura de análisis a través de la propuesta de una ficha de análisis de las canciones y el establecimiento de unas categorías para la clasificación, asociación, archivo y utilización de todo este material didáctico.

\section{Bibliografía}

Hemsy de Gainza, V. (2002) Pedagogía Musical. Dos décadas de pensamiento y acción educativa. Buenos Aires: Lumen.

Platón (ed. 1998) Diálogos. IV La República. Introducción, traducción y notas de Conrado Eggers Lan. Madrid: Editorial Gredos. Libro III, 398c a 403c.

SÁnchez De Andrés, L. (2009) Música para un ideal. Pensamiento y actividad musical del Krausismo e Institucionismo españoles (1854-1936). Madrid: Sociedad Española de Musicología.

Murray Schafer, R. (1970) ... Cuando las palabras cantan. Buenos Aires: Ricordi.

Vega Sestelo, M. C. De la (2013) La canción escolar en España entre 1900 y 1936. Tesis doctoral no publicada. Director Dr. José M. ${ }^{a}$ Hernández Díaz. Salamanca: Universidad de Salamanca, Departamento de Teoría e Historia de la Educación.

Willems, E. (1976) La preparación musical de los más pequeños. Buenos Aires: Editorial Universitaria de Buenos Aires (1962, r. ${ }^{a}$ ed.).

\section{Referencias normativas}

España. Ministerio de Instrucción Pública y Bellas Artes. Real Decreto de 26 de octubre de I90I, autorizando al Ministro de Instrucción Pública y Bellas Artes para que en los presupuestos generales de su departamento, a partir del que se forme para el año i902, incluya las partidas necesarias, conforme a las disposiciones de este decreto, para el pago de las atenciones de personal y material de las Escuelas públicas de primera enseñanza. Gaceta de Madrid de 30 de octubre de I90I, n. ${ }^{\circ} 303,497-499$.

España. Jefatura del Estado. Ley de 17 de julio de 1945, sobre Educación Primaria. Boletín Oficial del Estado de i8 de julio de 1945, n. $^{\circ}$ 199, 385-416.

España. Jefatura del Estado. Ley I4/1970, de 4 de agosto, General de Educación y Financiamiento de la Reforma Educativa. Boletín Oficial del Estado de 6 de agosto de 1970, n. ${ }^{\circ}$ I87, I2525-12546.

España. JeFATURA DEL EsTAdo. Ley Orgánica I/1990, de 3 de octubre, de Ordenación General del Sistema Educativo. Boletín Oficial del Estado de 4 de octubre de 1990, n. $^{\circ} 238,28927-$ 28942. 
España. Jefatura del Estado. Ley Orgánica 2/2006, de 3 de mayo de Educación. Boletín Oficial del Estado de 4 de mayo de 2006, n. ${ }^{\circ}$ I06, I7158-17207.

España. Jefatura del Estado. Ley Orgánica 8/20I3, de 9 de diciembre, para la mejora de la calidad educativa. Boletín Oficial del Estado de io de diciembre de 2013, n. ${ }^{\circ} 295,97858-97921$.

España. Ministerio de Educación, Cultura y Deporte. Real Decreto I26/20I4, de 28 de febrero, por el que se establece el currículo básico de la Educación Primaria. Boletín Oficial del Estado de I de marzo de 20I4, n. ${ }^{\circ}$ 52, 19394-19420.

Castilla y León. Consejería De Educación. Orden EDU/519/20I4, de 17 de junio, por la que se establece el currículo y se regula la implantación, evaluación y desarrollo de la educación primaria en la Comunidad de Castilla y León. Boletín Oficial de Castilla y León de 20 de junio de 20I4, n. ${ }^{\circ}$ II7, 44I8I-44776. 